\title{
A Dynamic Three-Dimensional Covalent Organic Framework
}

$\underline{\text { Wei Wang }}^{1}$, Yun-Xiang Ma ${ }^{1}$, Zhi-Jun $\mathrm{Li}^{1}$, Tian-Qiong Ma ${ }^{1}$, Yue-Biao Zhang ${ }^{2}$

\author{
${ }^{1}$ State Key Laboratory Of Applied Organic Chemistry, Lanzhou University, Lanzhou, China, ${ }^{2}$ School of Physical Science and \\ Technology, ShanghaiTech University, Shanghai, China \\ E-mail: wang_wei@lzu.edu.cn
}

The design, synthesis, and properties of dynamic porous crystals have been the subject of intense recent interest. Covalent Organic Frameworks (COFs) are a new type of crystalline porous organic materials. Recently, we achieved the construction of a new 3D crystalline covalent organic framework (COF) which displayed reversible structural transformation upon guest inclusion/release. The dynamic nature was demonstrated by in-situ 129Xe NMR spectroscopy, PXRD analysis, and vapor adsorption isotherms. In addition, this flexible COF showed potential application in heterogeneous catalysis. We expect that our results will promote further development of 3D dynamic crystalline COFs.

[1] Horike, S.; Shimomura, S.; Kitagawa, S. (2009). Nat. Chem. 1, 695.

[2] Côté, A. P.; et al. (2005). Science, 310, 1166.

[3] Ding, S.-Y.; Wang, W. (2013). Chem. Soc. Rev. 42, 548.

Keywords: Covalent Organic Framework, Dynamic porous crystals 